\title{
Exploring the Tradeoff between Performance and Overhead of the Relay Selection Mechanism in the Uniform Stress Routing Protocol
}

\author{
Nawel Alioua \\ Hitachi, Ltd. Research \& Development Group \\ Tokyo, Japan \\ nawel.alioua.th@hitachi.com
}

\author{
Haruo Takeda \\ Hitachi, Ltd. Research \& Development Group \\ Tokyo, Japan
}

\begin{abstract}
The constrained nature of Low power and Lossy Networks (LLNs) inevitably impacts fundamental networking tasks. The focus of our work is routing protocols, where the constraints of the network discourage the maintenance of large routing tables and the use of excessive control traffic, nonetheless optimizing for metrics such as delay and packet loss. In a previous work, we briefly introduced the Uniform Stress Routing protocol (USR), an approach inspired from the load distribution in trees. USR selects a limited set of relays at every node for all traffic forwarding, based on their ability to deliver traffic to the most solicited destinations, thus traffic directed to least solicited destinations will take suboptimal paths. This paper aims at further clarifying the concept of USR, then putting its performance to the test on Network Simulator 3. Experiments were conducted using the LR-WPAN module, and showed that USR allows a $13 \%$ decrease in control traffic, with no effect on the packet loss, but an increase in delays of $20 \%$.
\end{abstract}

\section{CCS CONCEPTS}

Network protocol design • Routing protocols • Logical / virtual topologies

\section{KEYWORDS}

Internet of things, routing in LLNs, biomimicry, topology optimization

\section{ACM Reference format:}

Nawel Alioua and Haruo Takeda. 2018. Exploring the Tradeoff between Performance and Overhead of the Relay Selection Mechanism in the Uniform Stress Routing Protocol. In Proceedings of the $14^{\text {th }}$ Asian Internet Engineering Conference (AINTEC'18). ACM, New York, NY, USA, 8 pages. https://doi.org/10.1145/3289166.3289168

Permission to make digital or hard copies of all or part of this work for personal or classroom use is granted without fee provided that copies are not made or distributed for profit or commercial advantage and that copies bear this notice and the full citation on the first page. Copyrights for components of this work owned by others than ACM must be honored. Abstracting with credit is permitted. To copy otherwise, or republish, to post on servers or to redistribute to lists, requires prior specific permission and/or a fee.

Request permissions from Permissions@acm.org.

AINTEC '18, November 12-14, 2018, Bangkok, Thailand

(C) 2018 Association for Computing Machinery.

ACM ISBN 978-1-4503-6131-6/18/11 ..\$15.00

https://doi.org/10.1145/3289166.3289168

\section{INTRODUCTION}

The Internet of Things (IoT) paradigm promises to have a serious impact on our everyday lives through the introduction of intelligence to the smallest piece of equipment we own [11]. The network formed by the intelligent "things" is constrained, and inevitably influences the way we design routing protocols. As stated in [4], "the limited available memory reduces the number of routes that a device can store; radio transmissions should be used as little as possible because of energy considerations, which also impose a reduction in routing control overhead."

In such constrained networking settings, it is interesting to turn for inspiration to the oldest and most constrained environment we know: nature. Biomimicry is a new discipline that aims at transposing nature's principles to help solve our problems in a novel interdisciplinary and innovative way [7]. Perhaps the most famous examples of biomimicry in Computer Science concern artificial intelligence, with neural networks, genetic algorithms, or swarm optimization methods. Particularly, an interesting survey about biomimicry in networking can be found in [8].

Our approach to the problem of routing in LLNs (Low Power and Lossy Networks) was derived from one of the least studied natural principles for applications in computer science, i.e. the uniform stress distribution in load-bearing structures, such as trees. These natural topologies dynamically adapt their shapes depending on the load they are subject to, resulting in optimized structures. To make the stress on the tree uniform, the most loaded zones are reinforced, and both the main load of heavy branches, and lighter load of thinner ones, are "routed" to the ground through these few reinforced zones. In routing terms, this translates into the selection of preferred relays by way of some reinforcement mechanism that favors the frequent destinations, and the utilization of these relays for both frequently sought after and rarely needed ones. This implies a simplification of route discovery and maintenance (since only few relays are used) by way of a diminished overhead, but at the cost of efficiency, since the preferred relays are suboptimal forwarding options for rarest traffic. The question driving the present paper is: to what extent does the routing protocol face a 
tradeoff between performance and the discovery and maintenance overhead?

The remainder of this paper is structured as follows. Section 2 gives a brief overview of routing in LLNs, followed by a proposed formulation of routing as a uniform stress distribution problem in Section 3. The following section presents the resulting routing protocol, before Section 5 details the conducted experiments.

\section{PREVIOUS WORKS ON ROUTING FOR LLNS}

The topic of routing protocols in the context of LLNs (Low Power and Lossy Networks) has attracted considerable attention. Researchers first looked into the existing pool of routing protocols developed for conventional networks, in addition to those targeting the closest relatives of LLNs: MANETs (Mobile Ad hoc NETworks). A study by the IETF ROLL (Routing Over Low power and Lossy networks) [1] concludes on the inadequacy of the considered protocols, and therefore calls for a special effort to accommodate the constraints of LLNs. Efforts followed, and RPL (IPv6 Routing Protocol for Low-Power and Lossy Networks) was developed and specified in RFC 6550 [2]. RPL operates by creating a DODAG (Destination Oriented Directed Acyclic Graph) which is a logical routing topology, where every node selects a parent according to pre-specified conditions. RPL supports three types of traffic patterns, namely, P2MP (point-to-multipoint), MP2P (multipoint-to-point) and P2P (point-to-point), although experiments show the inequality of this support and a particular inadequacy for P2P traffic [12].

RPL falls into the proactive category of routing protocols. A proactive protocol begins the process of route discovery before routes are actually needed. Routes being readily available by the time an application starts transmitting allows a faster traffic initiation. However, there is no prior knowledge about whether the routing information gathered in a proactive way, or in the case of RPL, the DODAG structure, will be of practical use in the future, which ultimately may constitute excessive processing for the LLN.

The second category of routing protocols is the reactive, where the route discovery process in only initiated when a route to a given destination becomes necessary. This gives the guarantee that the routes being discovered are practically needed, but on the other hand, the on-demand route discovery implies a delay before the first packets can be sent. Examples of such protocols include LOADng (The 6LoWPAN Ad hoc Routing Protocol next generation) [13] which is a simplified version of the ancestor AODV (The Ad hoc On-Demand Distance Vector) routing protocol [14]. Both of these protocols are based on three main operations: route discovery, route maintenance and local connectivity management, using the following control messages: RREQ (Route REQuest), RREP (RouteREPly) and RERR (Route ERRor), with the difference that these processes are simplified to reduce the control traffic in LOADng [13].

As bio-inspired routing protocols, AntNet [15] and AntHocNet [16] are famous examples. These protocols use the Ant Colony Optimization method for routing, and are based on the concept of stigmergy, where ant agents communicate by modifying their environment. Both protocols are proactive, and periodically send ant agents to collect routing information toward randomly chosen destinations.

\section{ROUTING AS A UNIFORM STRESS DISTRIBUTION PROBLEM}

Uniform stress in nature can be observed in organisms such as trees. It is achieved by maintaining a fair distribution of the load that the structure is subject to, by adding material around the most loaded areas as in trees and bones, and removing materials from the least loaded areas as in bones. This simple yet powerful design rule is referred to as "the axiom of uniform stress" [3], and was originally applied for the durable design of mechanical parts $[9,10]$.

\subsection{Uniform Stress Distribution in Natural Structures and Constrained Routing}

The constrained nature of LLNs in terms of technical capabilities, such as memory, energy and transmission range, and the goal of establishing routing topologies, seem to constitute enough similarities to motivate a closer look at the natural structures and their adaptive stress distribution. We further identify the following analogies:

Table 1: Analogies between physical structures in nature and constrained networks

\begin{tabular}{|l|l|l|}
\cline { 2 - 4 } \multicolumn{1}{c|}{} & Physical Structures & Constrained Networks \\
\hline $\begin{array}{l}\text { Structure } \\
\text { connections }\end{array}$ & $\begin{array}{l}\text { Unit composed of } \\
\text { smaller parts (from } \\
\text { branches down to } \\
\text { particles) linked } \\
\text { together to form a } \\
\text { certain shape. }\end{array}$ & $\begin{array}{l}\text { Unit composed of } \\
\text { communication-capable } \\
\text { devices, linked together } \\
\text { by data traffic. }\end{array}$ \\
\hline Stress & $\begin{array}{l}\text { * The reactive force } \\
\text { of the structure in } \\
\text { response to the load } \\
\text { applied on it in real } \\
\text { environment. } \\
* \text { A force has } \\
\text { intensity and } \\
\text { direction. }\end{array}$ & $\begin{array}{l}\text { The reactive topology } \\
\text { creation in response to all } \\
\text { the traffic flows between } \\
\text { pairs of nodes. } \\
\text { the traffic flow has an } \\
\text { intensity the amount of } \\
\text { data) and a direction } \\
\text { from source to } \\
\text { destination) }\end{array}$ \\
\hline $\begin{array}{l}\text { Supporting the } \\
\text { stress }\end{array}$ & $\begin{array}{l}* \text { Conservation of } \\
\text { unity and stability (no } \\
\text { breakage) }\end{array}$ & $\begin{array}{l}* \text { Conservation of } \\
\text { connectivity and response } \\
\text { to fluctuations (link } \\
\text { failure) }\end{array}$ \\
\hline
\end{tabular}


Exploring the Tradeoff between Performance and Overhead of the Relay Selection Mechanism in the Uniform Stress Routing Protocol
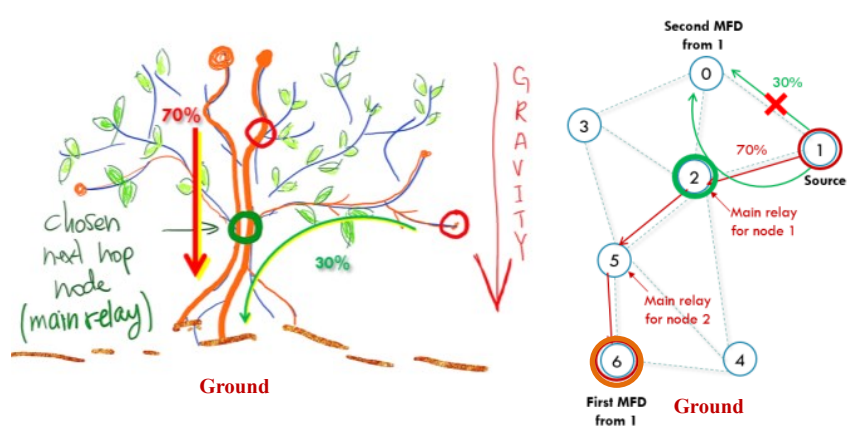

Figure 1: Routing through a main relay

\begin{tabular}{|l|l|l|}
\hline \multirow{2}{*}{ Optimization } & $\begin{array}{l}\text { To achieve a } \\
\text { maximum strength } \\
\text { using a minimum } \\
\text { amount of material }\end{array}$ & $\begin{array}{l}\text { To achieve a maximum } \\
\text { delivery rate with a } \\
\text { minimum delay using the } \\
\text { least amount of resources. }\end{array}$ \\
\hline
\end{tabular}

If we were to design a routing method based on the analogies above, then the amount of traffic circulating in the network will be the equivalent of the load, i.e. the main determinant of the routing decision. Therefore, just as the total load borne by a tree is composed of main loads and secondary loads (the main load being the one borne by the trunk), the total traffic circulating through a single router could show a main traffic (to one or more heavily solicited destinations) and secondary traffic, as depicted in Figure 1. We define the most frequent destination (MFD) at the level of a node as the destination to which the majority of traffic is routed. That traffic we refer to as the most frequent traffic. Each router is the trunk of a (logical) routing topology. The entire network is the superposition of such topologies.

The key observation is that in trees, the secondary load adds up to the main load and gather in thicker branches, recursively relaying it down to the trunk. Replicating this observation in constrained networks would mean designating, at every router, main relay nodes for transmitting both the main flows as well as the secondary ones. These main relay nodes are designated as to accommodate the MFD(s). This would limit the amount of routes that should be maintained by the router. In fact, keeping updated information about a destination would require the router to send frequent RREQ messages, which means a higher energy consumption and an increased risk for collisions. Therefore, maintaining a limited number of relays saves "material", i.e. resources (energy and bandwidth) in the network. Nonetheless, the main relays chosen at a router might represent suboptimal choices in terms of delay for least frequent destinations.

\section{USR: THE UNIFORM STRESS ROUTING PROTOCOL}

AINTEC '18, November 12-14, 2018, Bangkok, Thailand

As our objective is to limit the control traffic and the processing to the minimum required by the application layer, USR [17] operates as a reactive routing protocol. It mainly relies on the following mechanisms:

\subsection{The Route Discovery Process}

The route discovery process is similar to LOADng's when it comes to the exchange of multicast RREQs and unicast RREPs. When a route is needed at a source node, a RREQ message is multicast, then relayed by all the neighbors till it reaches the intended destination. There, a RREP message can be formed containing, most importantly, the arrival time of the request to allow the source to calculate the one-way delay. Note that the destination is the only node allowed to reply to RREQs, even if intermediate nodes have the desired routing information. The RREP travels back in a unicast fashion toward the source, while updating the metrics related to this destination. In our initial design, the metric we consider is the average delay to the destination $j$ through neighbor $i$, denoted $A D_{i j}$, and updated upon reception of a RREP as follows:

$$
A D_{i j}(t)=\alpha A D_{i j}(t)+(1-\alpha) a d_{i j}(t)
$$

$a d_{i j}(t)$ being the new incoming value of $A D_{i j}$. To keep track of the destinations searched by a node, corresponding entries are stored in the Stress Table, along with the obtained $A D_{i j}$ values, and a status that can be "Waiting", "Answered", or "Unreachable". The obtained average delays will serve to compute the reinforcement value that will be the selection basis of the preferred relay(s).

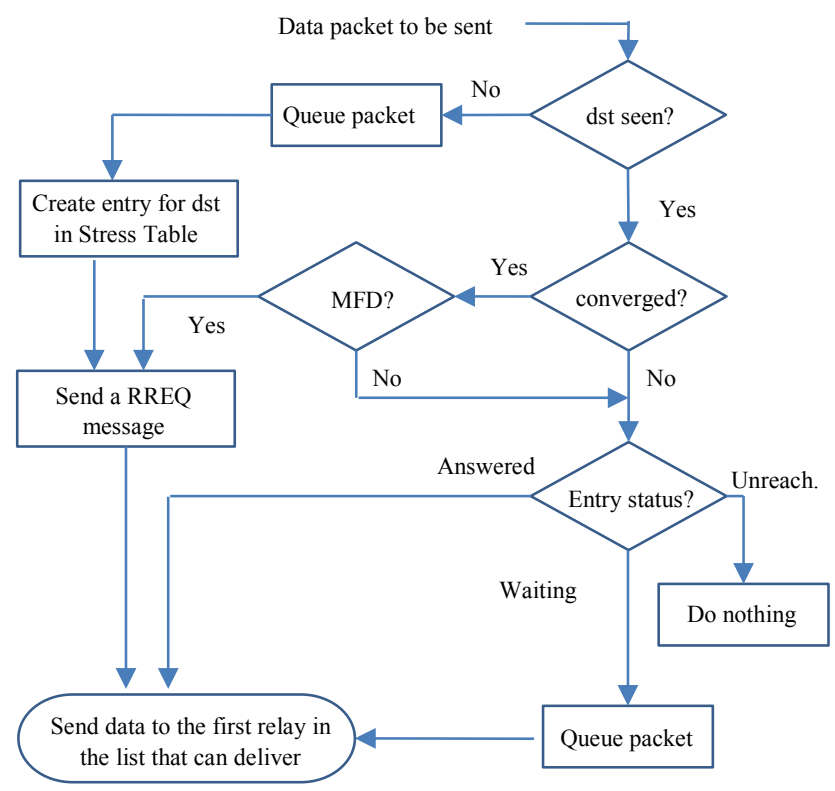

Figure 2: Simplified diagram of the forwarding process in USR

Since the metric used in this implementation is the delay, the reinforcement value, we call here the Uniform Delay $\left(U D_{i}\right)$ for each neighbor $i$, is expressed as:

$$
U D_{i}=p_{1} * A D_{i 1}+p_{2} * A D_{i 2}+\cdots+p_{n} * A D_{i n}
$$


$p_{j}$ being the percentage of traffic directed to destination $j$, gives more importance to the delay obtained for the MFD(s).

However, an important difference with traditional reactive route discovery is that USR defines a convergence state, in which RREQs are only sent to $\mathrm{MFD}(\mathrm{s})$ to keep the routing information updated, or new destinations. A simplified diagram of the forwarding process is presented in Figure 2.

\subsection{The Relay Selection Mechanism}

After the route discovery process is completed, the neighbors are sorted by descending order of their $U D$. A packet to be forwarded will take the first relay $i$ in the sorted list that has proven in the past a successful delivery to the related destination $j\left(A D_{i j} \neq \infty\right)$.

\section{TESTS AND RESULTS}

In this evaluation, we focus our attention on the effect of one feature of USR, that is the forwarding through preferred relays. To serve as a basis for comparison, we define a variant of USR that uses the full information available in the stress table about the best next hop nodes for each destination. This variant, here denoted "USR Full", differs from USR in the following two ways:

1. A packet is always forwarded to the best known relay for the destination sought after, while USR aggregates the forwarding to different destinations through the top preferred relays.

2. Therefore, to keep a fair routing information update across all destinations, a new request is sent to every destination whose validity has expired, whereas USR only requests recent information from destinations belonging to the MFD set.

\subsection{Experimental Settings}

We conducted experiments on Network Simulator 3 (ns-3.27 release) under a Linux system running on an Intel Xeon 8-core CPU, with 16 GB of RAM.

Since this research aims at constrained networks, the simulation uses the Low-Rate, Wireless Personal Area Network (LR-WPAN) ${ }^{1}$ ns-3 module, which implements the IEEE 802.15.4 MAC (Medium Access Control) and PHY (physical layer) standards.

The simulated network consists in randomly placed nodes, generated using the unit disk graph model [6], a practical way of representing wireless network connectivity that would provide, in addition, a graph-theoretic basis for further analysis. Unit disk graphs are the intersection graphs of equal sized circles of radius $r$, which in the context of wireless networks is the node's range of transmission.

Another parameter turned out to be crucial for the generation of random yet practical networks using LR-WPAN, i.e. the node degree denoted $d$. In fact, a completely random unit disk graph model can produce highly dense networks, and our trial-and-error cycles show that if a node has a degree of 5 or more, all of the data flows involving it will experience extremely high packet loss rates.

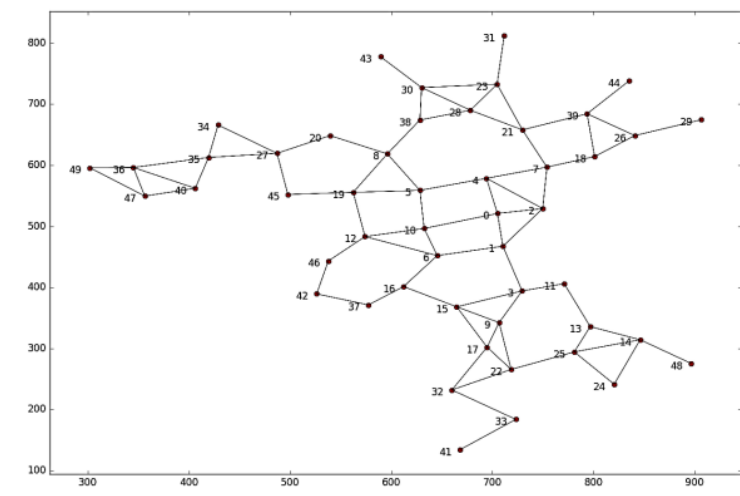

Figure 3: The simulation network

As this is only an empirical observation, further investigation would be highly valuable for the future developments of LRWPAN simulations. For our practical purpose, we set the maximum node degree to $d=4$.

Figure 3 shows the network generated for these experiments, composed of $n=50$ routers with radius $r=80 \mathrm{~m}$, and node degree $d=4$.

Once the nodes are placed on the simulation plane, we generate the traffic patterns. Each execution round will have $S$ simultaneous traffic flows, whose extremities are designated at random. Traffic flows are identical: 100 packets are sent at a frequency of one packet per second.

\subsection{Results and Discussion}

The first series of experiments consists of 45 executions, with an increasing number of simultaneous traffic flows involving different pairs of nodes. For each execution with a given number of flows, however, the traffic is exchanged between the same pairs in USR, and USR Full.

The results of the first series are summarized in Figure 4 and 5. where the horizontal axis is the number of simultaneous traffic flows in each execution.

Figure 4 shows the number of requests sent from, and the number of replies received at the originator of the data flow. The "route discovery ratio" is the ratio of the two previous values. It simply informs about the average number of replies received for each request sent.

As expected, the number of requests sent in the case of USR is inferior to that of USR Full. Indeed, once USR converges (for the sake of an early evaluation, once a timer has expired), the only requests allowed are the ones targeting MFDs. This incurs an average drop of $12 \%$ on the total control traffic.

Consequently, USR Full obtains more replies although the fluctuation of this metric is important, and does not seem to be related to the sheer number of traffic flows. USR Full, being more generous in terms of request messages, is more likely to cause collisions, the result of which in terms of control traffic is visible through the route discovery ratio. This metric being predominantly

\footnotetext{
${ }^{1}$ https://www.nsnam.org/docs/models/html/lr-wpan.html
} 
Exploring the Tradeoff between Performance and Overhead of the Relay Selection Mechanism in the Uniform Stress Routing Protocol

higher in USR, is a sign that more request messages do not necessarily yield a better route discovery. This gain in terms of control traffic did not affect the total data packet loss for most of the executions, as shown in Figure 5. However, it is worth noting that the reason why the packets are lost is fundamentally different in the two variants. The difference in the number of packets lost after transmission (not received) is always negative, which means that USR loses more packets after transmission, most likely caused by overloading the topmost preferred relays. On the other hand, the difference in the number of packets that are never sent in the first place is always positive, implying that USR Full is worse at putting packet onto the physical medium. Knowing that the only reason that might cause a packet to never be sent in this particular implementation is to be cleared away from the routing protocol queue, we naturally relate to the previous route discovery ratio, and further deduce that the packets must have been dropped from the queue before the replies reached the originator of the request.
Figure 5 also shows the average one-way delay of both variants. Unsurprisingly, USR causes a higher delay for most executions, $32 \%$ more on average than USR Full. This is due to the fact that, by definition, USR sends the traffic aiming at least solicited destinations through the first relay from the ordered neighbor list that is able to reach the said destination, which means suboptimal relays for relatively non-majoritarian traffic.

Again, a common observation from the results above is the relatively high fluctuation of the metrics under a completely random, thus uncontrolled, selection of the pairs constituting the traffic load of the network. As a summary of the above results, the median value of the gain in control traffic of $13 \%$ corresponds to a delay increase of about $20 \%$.

In an attempt to shed some light on the fluctuation issue, we conduct a second series of experiments on the same randomly generated network with a reduced number of 12 random traffic
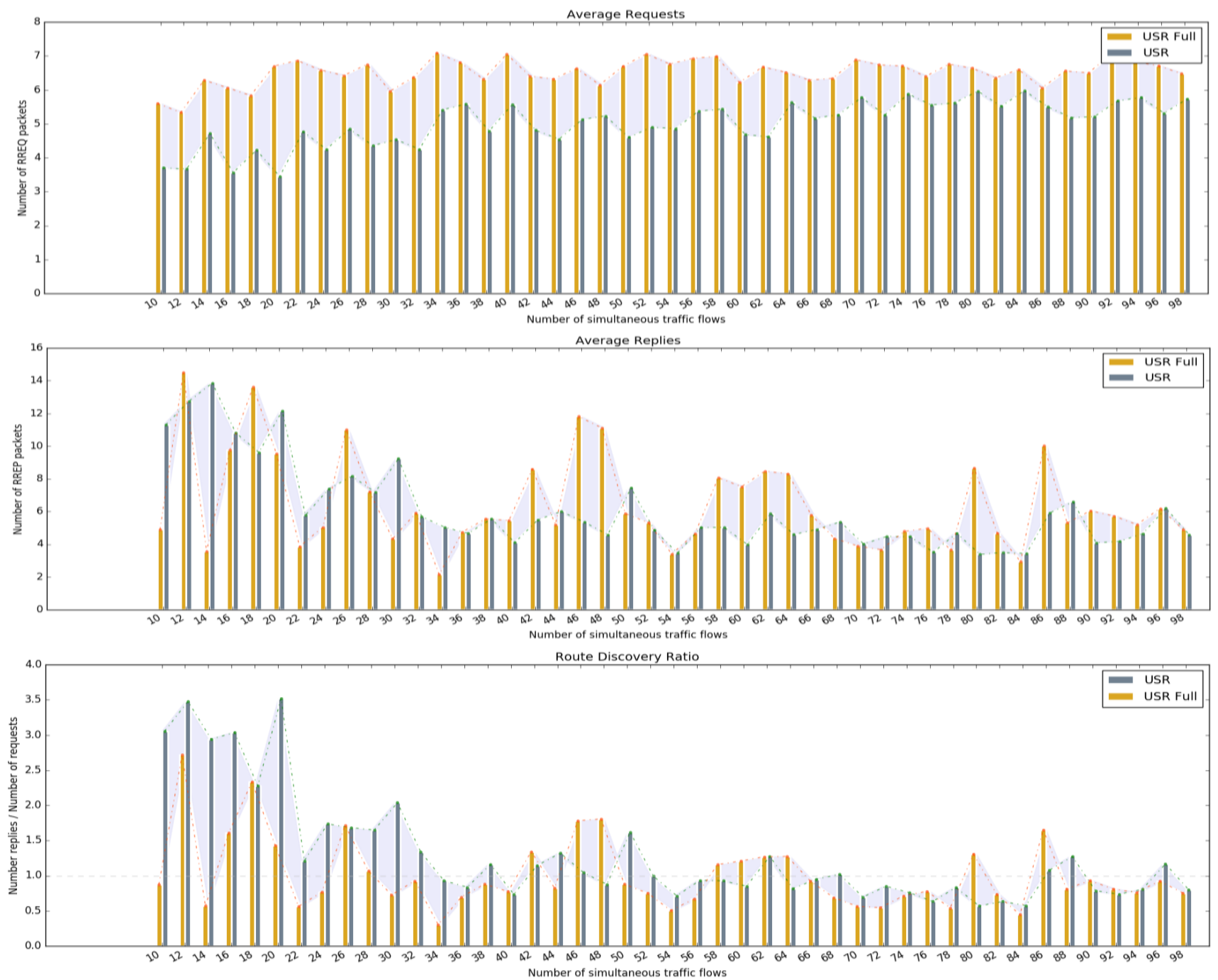

Figure 4: Average amount of control traffic 

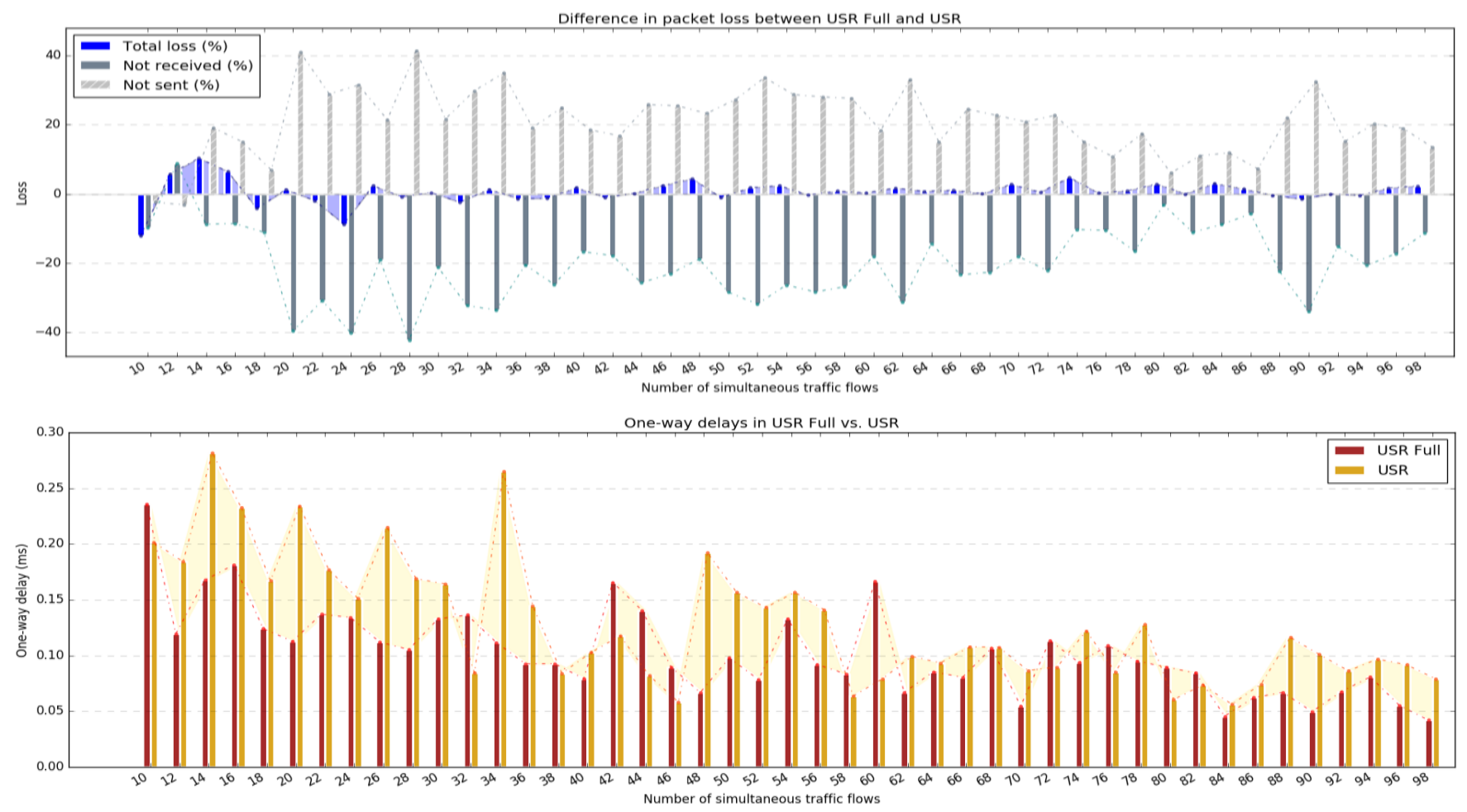

Figure 5: Average packet loss and one-way delays

flows, but with a controlled hop count. Table 2 shows the involved node identifiers and the hop count separating each pair.

Table 2: Pairs and hop counts in the second experiment series

\begin{tabular}{|cc||cc|}
\hline Pairs & Hop count & Pairs & Hop count \\
\hline $\mathbf{1 7 - 3 2}$ & 1 & $\mathbf{1 4 - 2 1}$ & 7 \\
\hline $\mathbf{3 - 1 7}$ & 2 & $\mathbf{0 - 4 7}$ & 8 \\
\hline $\mathbf{1 8 - 2 8}$ & 3 & $\mathbf{2 4 - 2 6}$ & 9 \\
\hline $\mathbf{3 - 1 2}$ & 4 & $\mathbf{4 8 - 3 8}$ & 10 \\
\hline $\mathbf{7 - 2 2}$ & 5 & $\mathbf{3 2 - 4 0}$ & 11 \\
\hline $\mathbf{3 7 - 2 0}$ & 6 & $\mathbf{4 8 - 4 9}$ & 13 \\
\hline
\end{tabular}

The simulation is first run with only one active pair at a time, then with all 12 communicating pairs simultaneously. This allows us to separate the effect of two important factors:

1. The quality of the route selected in each protocol. Running one traffic flow at a time informs us about the empirical upper bound for the performance metrics.

2. The collisions caused by the simultaneous transmissions. The results obtained in the simultaneous scenario for each protocol are divided by their respective counterparts obtained from the one-pair-at-a-time executions. This allows to assess the resilience of each variant of the routing protocol to traffic load.
In Figure 7 below, the packet loss increases as the number of hops increase, with a few exceptions. In USR, the pairs with hop counts of 8,11 and 13 obtain an unexpectedly low loss. A quick look at the actual position of these pairs in the simulation network (Figure 6) shows that, although these paths are relatively long, important parts of the paths are located in the periphery of the network, whereas pairs 10 and 7 suffering a higher loss, include highly dense areas. This is a direct consequence of the multicasting of RREQ messages, where the more dense the network is, and the more RREQ messages will be issued. The density of the network does not play in favor of USR Full either, since the RREQ

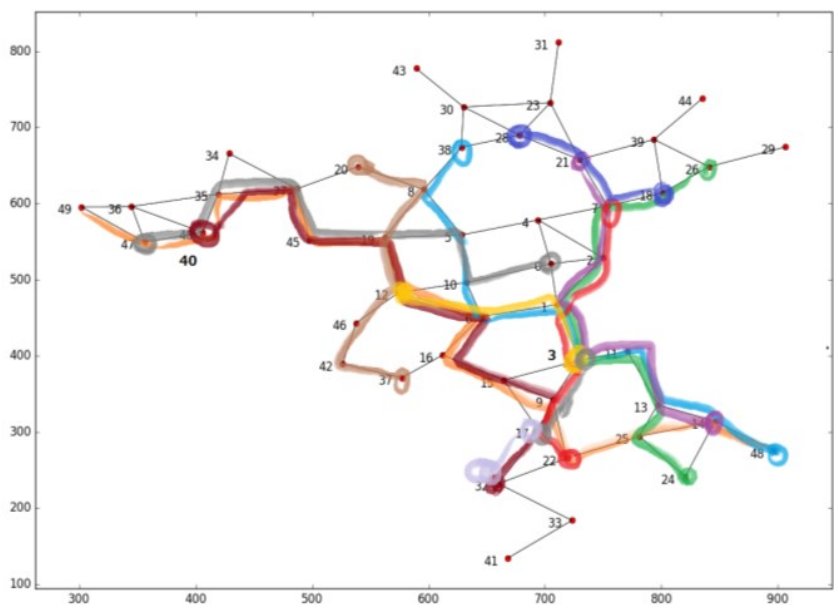

Figure 6: the traffic flows of the second series of experiments 
Exploring the Tradeoff between Performance and Overhead of the Relay Selection Mechanism in the Uniform Stress Routing Protocol

mechanism is the same as USR, with the addition that it is not limited by any convergence timer. This causes the loss to be high even for very short paths.

The ratio of the simultaneous execution loss over that of the isolated pairs in each variant shows a clear divergence, nonetheless replicating the observation from the packet loss in Figure 5. USR tends to loose packets between pairs at 1,2,3, and 4 hops distance. The locations of these pairs in the network makes them ideal traffic aggregators and if several of their neighbors select them as preferred relays, collision occurrence will be higher in these areas. As for USR Full, the notable difference is that the number of unsent packets (dropped from the protocol queue) peaks on all paths longer than 5 hops, as a reminder that "less is more" in terms of control traffic.

Figure 8 represents the delay values obtained from the same experiments. Confirming the results of Figure 5, USR is less resilient in terms of delay than USR Full, due to the traffic aggregation on the preferred relays. Nonetheless, in USR Full, pair 24-26, with a hop count of 9, is an exception to the trend, where the path is traversed by several other flows, making the congestion from USR Full control traffic cause longer delays.

\section{CONCLUSION AND FUTURE WORK}

This paper presented the Uniform Stress Routing Protocol (USR), a reactive routing protocol targeting constrained networks, and inspired from the load distribution mechanism of natural
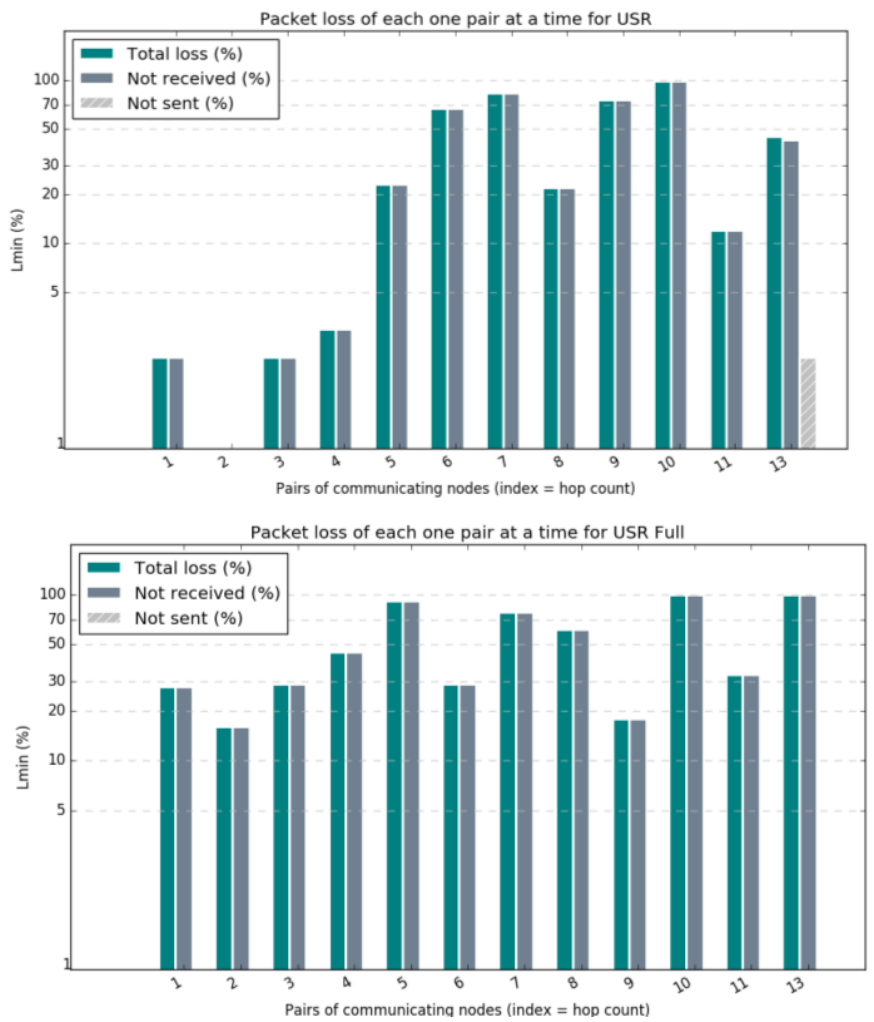

AINTEC '18, November 12-14, 2018, Bangkok, Thailand
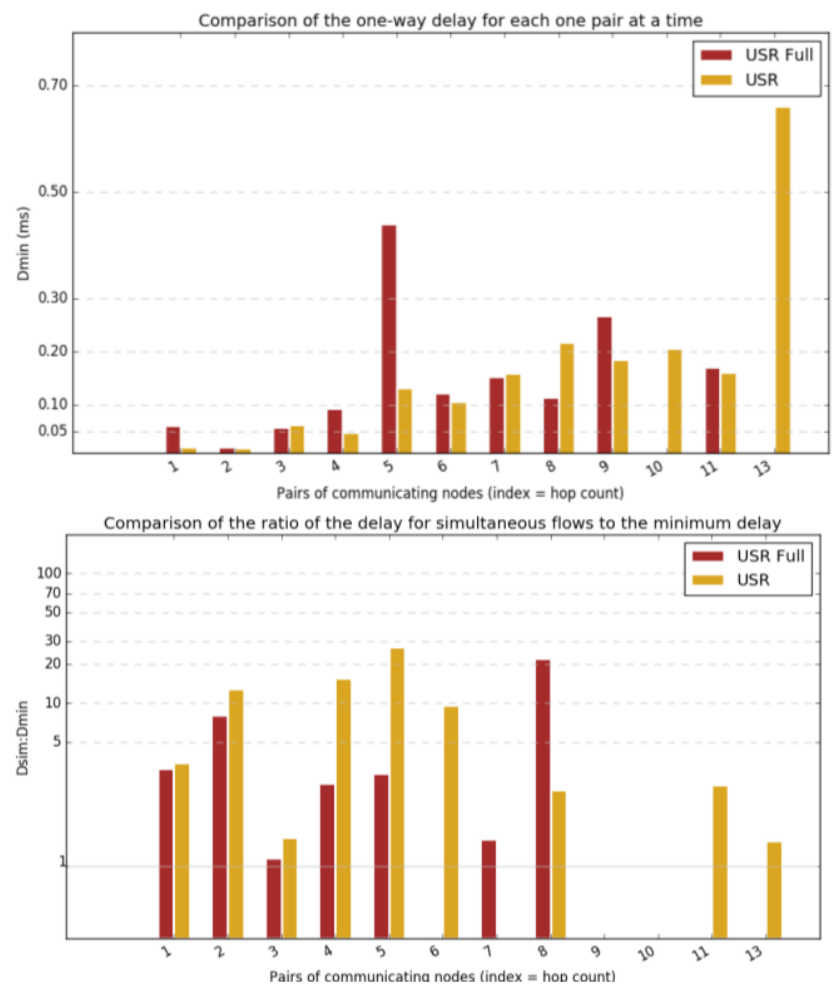

Figure 8: Average delays from individual pairs vs. simultaneous execution
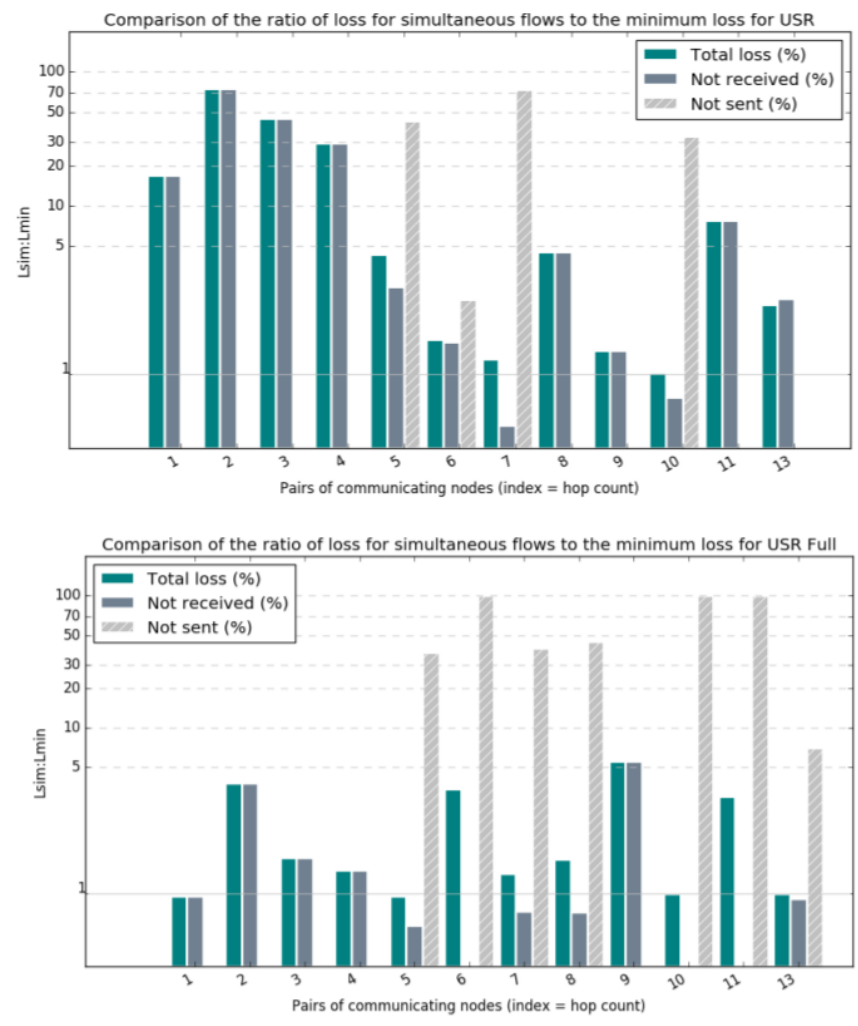

Figure 7: Average packet loss from individual pairs vs. simultaneous execution 
constrained structures such as trees. The protocol is still at its early stages of design and development, which positions this work at an intersection point between conceptual bio-inspired design, and the practical challenges and technicalities of routing protocols development and simulation for LLNs.

The experiments conducted at this stage aimed at comparing our early version of USR with a variant that inhibits one of its key features: the forwarding of traffic through the preferred relays. In USR, preferred relay(s) are selected both for the MFDs, for which the relays are optimal, and for non-majoritarian traffic, the paths through which are inevitably sub-optimal. USR Full, the variant used for comparison, always selects the relay presenting the optimal performance, regardless of how frequently the destination is sought after. The results show that the forwarding through the preferred relay(s) in USR achieve a 13\% drop in control traffic with no significant effect on the overall packet loss, but a delay increase of $20 \%$. These results motivate further refinement, both on the conceptual level and the purely technical one.

Moreover, our simulations using the LR-WPAN module on ns-3 hints at the importance of the following factors, and calls for more sophisticated testing allowing more visibility and control:

- $\quad$ The collision rate in IEEE 802.15.4 technology, particularly in LR-WPAN, the related simulation module in ns-3.

- The influence of the node degree on the medium access control, and on the routing protocol's relay selection mechanism.

Future work on USR includes:

- Diversifying the metrics used for ordering the neighbors and selecting the relay. In the present version of the protocol, the only metric used is the delay from the source to the destination. Other metrics include the hop count and the ETX (Expected Transmission Count).

- Refining the condition of convergence. The protocol should ultimately be able to dynamically detect a stabilization of the traffic pattern to switch to the converged state. Conversely, the minimum amount of pattern variation that triggers a switch back to non-converged state should also be dynamically defined.

- A more complete comparative study with previous routing protocols for LLNs. This requires either a simulation platform migration, as no such protocols are implemented in ns-3, or a hardware-based implementation.

\section{ACKNOWLEDGMENTS}

The authors would like to thank Renato Miyagusuku from the University of Tokyo, and Jairo López and Jens Dönhoff from Hitachi Ltd. R\&D Group, for their insightful comments and useful recommendations.

\section{REFERENCES}

[1] P. Levis, A. Tavakoli S. Dawson-Haggerty (2009). Overview of Existing Routing Protocols for Low Power and Lossy Networks. https://tools.ietf.org/html/draft-ietf-roll-protocols-survey-07.

[2] T. Winter, P. Thubert, A. Brandt, J. Hui, R. Kelsey, P. Levis, K. Pister, R. Struik, JP. Vasseur, R. Alexande (2012). RPL: IPv6 Routing Protocol for Low-Power and Lossy Networks. RFC 6550.

[3] C. Mattheck.1998. Design in Nature: Learning from Trees. SpringerVerlag Berlin Heidelberg.

[4] Vucinic, M., B. Tourancheau, and A. Duda (2013). Performance Comparison of the RPL and LOADng Routing Protocols in a Home Automation Scenario. 2013 IEEE Wireless Communications and Networking Conference (WCNC), 1974-79.

[5] Yi, Jiazi, T. Clausen, and U. Herberg (2015). Depth-First Forwarding for Unreliable Networks: Extensions and Applications. IEEE Internet of Things Journal 2 (3), 199-209.

[6] B.N. Clark, C.J. Colbourn and D.S. Johnson (1990). Unit Disk Graphs. Discrete Mathematics 86, 165-177

[7] Janine Benyus. 1997. Biomimicry: Innovation Inspired by Nature. William Morrow, 1997.

[8] F. Dressler, and B. Akan (2010). A Survey on Bio-Inspired Networking. Computer Networks, New Network Paradigms, 54 (6): 881-900.

[9] A. Baumgartner, S. Burkhardt, C. Mattheck. (1991). The KILLOPTION - A powerful method to prepare engineering low weight design proposals. Mechanical behaviour of materials - VI; Proceedings of the 6th International Conference, Kyoto, Japan. Vol. 4 (A93-40776 16-39), p. 639-642.

[10] C. Mattheck, S. Burkhardt (1991). Shape Optimization of Engineering Components by Adaptive Biological Growth. In Engineering Optimization in Design Processes, edited by Hans A. Eschenauer, Claus Mattheck, and Niels Olhoff, 15-24. Lecture Notes in Engineering 63. Springer Berlin Heidelberg.

[11] L. Atzori, A. Iera, and G. Morabito (2010). The Internet of Things: A Survey. Computer Networks 54 (15): 2787-2805.

[12] T. Clausen, U. Herberg, M. Philipp (2011). A Critical Evaluation of the IPv6 Routing Protocol for Low Power and Lossy Networks (RPL). IEEE 7th International Conference on Wireless and Mobile Computing, Networking and Communications (WiMob). 365-72.

[13] T. Clausen,Y. Jiazi, and A.C. de Verdiere (2012). LOADng: Towards AODV Version 2. IEEE Vehicular Technology Conference (VTC Fall), 1-5.

[14] C. Perkins, E. Belding-Royer, and S. Das (2003). Ad hoc On-Demand Distance Vector (AODV) Routing. RFC 3561.

[15] G. Di Caro and M. Dorigo (1998). AntNet: Distributed Stigmergetic Control for Communications Networks. J. Artif. Int. Res., vol. 9, no. 1. 317-365.

[16] G. Di Caro, F. Ducatelle, and L. M. Gambardella (2004). AntHocNet: An Ant-Based Hybrid Routing Algorithm for Mobile Ad Hoc Networks. Parallel Problem Solving from Nature - PPSN VIII, X. Yao, E. K. Burke, J. A. Lozano, J. Smith, J. J. Merelo-Guervós, J. A. Bullinaria, J. E. Rowe, P. Tiňo, A. Kabán, and H.-P. Schwefel, Eds. Springer Berlin Heidelberg, pp. 461-470.

[17] N. Alioua, K. Nakamura, M. Kamio, N. Koshizuka and K. Sakamura (2016). USR: Uniform Stress Routing Protocol for Constrained Networks. IEEE 5th Global Conference on Consumer Electronics (GCCE). 1-3. 